EPJ Web of Conferences 60, 16003 (2013)

DOI: $10.1051 /$ epjconf $/ 20136016003$

(C) Owned by the authors, published by EDP Sciences, 2013

\title{
Measurements of the production cross-section of top quark pairs using the ATLAS detector at the LHC
}

\author{
Kevin Finelli ${ }^{1, a}$ \\ On behalf of the ATLAS Collaboration \\ ${ }^{1}$ Duke University, Physics Department
}

\begin{abstract}
An overview of several ATLAS measurements of top quark pair production in proton-proton collisions at the LHC at center-of-mass energies of 7 and $8 \mathrm{TeV}$ is presented. Inclusive cross-section measurements of top quark pair production in various decay channels, analyses of differential cross-sections, studies of additional jet activity in top quark pair production, as well as an analysis of top quark pair production with associated heavy flavor jets are presented. All of the measurements shown are in good agreement with the latest Standard Model predictions.
\end{abstract}

\section{Introduction}

The Large Hadron Collider [1] (LHC) has delivered proton-proton collisions at center of mass energies of $\sqrt{s}=7 \mathrm{TeV}$ and $\sqrt{s}=8 \mathrm{TeV}$ during 2011 and 2012 . The energies and luminosities delivered have allowed the ATLAS experiment [2] to collect an unprecedented large sample of top quark pair $(t \bar{t})$ production events. This document describes seven recent measurements of the top quark pair production cross-section from the ATLAS experiment. Inclusive measurements of $\sigma_{t \bar{t}}$ are covered in the single lepton channel at $\sqrt{s}=8 \mathrm{TeV}$ (Section 2), in the hadronic tau plus jets channel at $\sqrt{s}=7 \mathrm{TeV}$ (Section 3), and in the hadronic tau plus lepton channel at $\sqrt{s}=7 \mathrm{TeV}$ (Section 4). A measurement of the production of $t \bar{t}$ events with additional heavy flavor is then presented (Section 5), followed by a measurement of $t \bar{t}$ production with a veto on additional central jet activity (Section 6). Also shown are differential cross-section measurements with respect to the $t \bar{t}$ invariant mass $\left(m_{t \bar{t}}\right)$, transverse momentum of the $t \bar{t}$ system $\left(p_{T, t \bar{t}}\right)$, and the $t \bar{t}$ rapidity $\left(y_{t \bar{t}}\right)$ (Section 7), and finally, a study of the production of $t \bar{t}$ in association with additional jets (Section 8) is shown.

Inclusive measurements provide a valuable test of Standard Model predictions using state of the art perturbative QCD calculations. NNLO cross-sections at $\sqrt{s}=$ $7 \mathrm{TeV}$ and $\sqrt{s}=8 \mathrm{TeV}$ are calculated to be [3]

$$
\begin{aligned}
\sigma_{t \bar{t}}^{7 \mathrm{TeV}} & =172.0_{-5.8}^{+4.4}(\text { scale })_{-2.8}^{+4.7}(\text { pdf }) \mathrm{pb} \\
\sigma_{t \bar{t}}^{8} \mathrm{TeV} & =245.8_{-8.4}^{+6.2}(\text { scale })_{-6.4}^{+6.2}(\text { pdf }) \mathrm{pb}
\end{aligned}
$$

where scale refers to uncertainties on the renormalization and factorization scales, and pdf refers to the uncertainty from parton distribution functions.

\footnotetext{
ae-mail: kevin.finelli@cern.ch
}

\section{Single lepton channel at $\sqrt{s}=8 \mathrm{TeV}$}

In the single lepton channel, one of the two $W$ bosons from the $t \bar{t}$ pair decays into light quarks which subsequently hadronize into jets, while the other $W$ boson decays to an electron, muon, or leptonically-decaying tau lepton together with the corresponding neutrino. This analysis [4] uses $5.8 \mathrm{fb}^{-1}$ of $\sqrt{s}=8 \mathrm{TeV}$ data and selects events with large missing transverse momentum $\left(\mathbb{E}_{T}\right)$, large $W$ transverse mass $\left(m_{T}(W)\right),{ }^{1}$ and one electron or muon with transverse momentum $p_{T}(\ell)>40 \mathrm{GeV}$. Furthermore, three or more jets with transverse momentum $p_{T}>25$ (jet) $\mathrm{GeV}$ are required, and one or more jet must be tagged as a $b$-jet candidate. The MV1 [5] algorithm at $70 \% b$-tagging efficiency operating point is used to identify jets arising from a $b$-quark. A likelihood discriminant fit is then employed to distinguish $t \bar{t}$ and $W+$ jets contributions and to determine their relative normalizations. The discriminant employed in the fit is constructed from the transformed aplanarity $\left(A^{\prime}=\exp -8 A\right)^{2}$ and lepton pseudorapidity, as shown in Figure 2. The cross-section is measured to be

$$
\sigma_{t \bar{t}}=241 \pm 2 \text { (stat.) } \pm 31 \text { (syst.) } \pm 9 \text { (lumi.) pb }
$$

The measurement is dominated by systematic uncertainty, the primary component of which is signal modeling uncertainty $(11 \%)$.

\footnotetext{
${ }^{1}$ For an event with a neutrino and charged lepton, the transverse mass is defined as $m_{T}(W)=\sqrt{2 p_{T}^{\ell} p_{T}^{v}\left(1-\cos \phi^{\ell}-\phi^{v}\right)}$, where $p_{T}$ is the transverse momentum, $\phi$ is the azimuthal angle, and $\ell$ and $v$ refer to the charged lepton and the neutrino, respectively.

${ }^{2}$ Aplanarity, $A$, is defined as $A=\frac{3}{2} \lambda_{3}, \lambda_{3}$ being the smallest eigenvalue of the normalized momentum tensor calculated using the momenta of all jets and the lepton.
} 

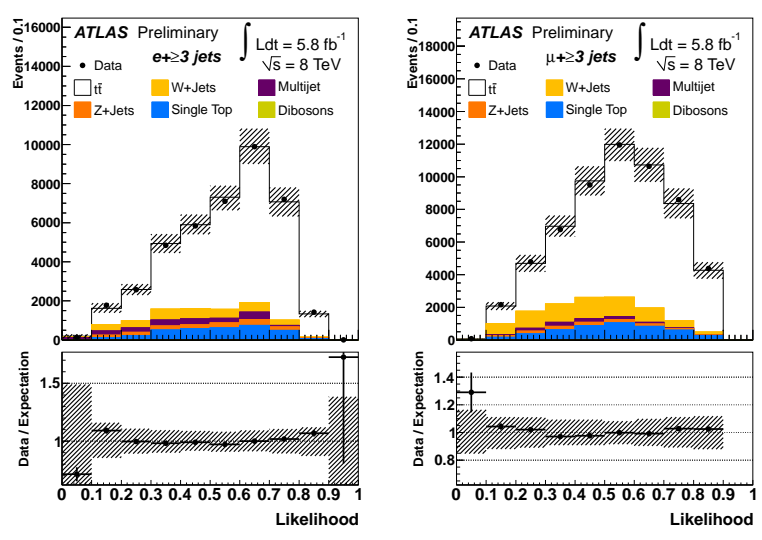

Figure 1. Fit to the resulting likelihood discriminant distribution, $D\left(\eta_{\ell}, A^{\prime}\right)$ in data in the $e+$ jets (left) and $\mu+$ jets (right) channels [4]. The hatched bands display the combined expected statistical and systematic uncertainty.

\section{Tau plus jets channel at $\sqrt{s}=7 \mathrm{TeV}$}

In the tau plus jets channel, one $W$ boson decays to light quarks that hadronize to jets, while the other decays to a tau neutrino and tau lepton, which subsequently decays hadronically. This channel allows a test of flavordependent effects in top quark decays as well as studies backgrounds in certain new physics searches, such as those predicting a charged Higgs boson decaying as $H^{ \pm} \rightarrow$ $\tau^{ \pm} v_{\tau}$. The ATLAS cross-section measurement in this channel [6] uses $1.67 \mathrm{fb}^{-1}$ of data taken at center of mass energy $\sqrt{s}=7 \mathrm{TeV}$. Events are selected with five or more jets with transverse momentum $p_{T}$ (jet) $>20 \mathrm{GeV}$, two or more of which must be $b$-tagged jets. This all-hadronic final state is selected using a dedicated high-level trigger $b$-tagging algorithm. A likelihood fit to the track multiplicity $\left(n_{\text {track }}\right)$ distribution is performed with three templates: the tau/electron template (containing signal event candidates), the gluon-jet template (mainly from multi-jet events), and quark-jet template (from background $t \bar{t}$ and $W+$ jets), shown in Figure 2. Backgrounds are estimated from Monte Carlo simulation and subtracted to extract the total number of signal events. The cross-section is then found to be

$$
\sigma_{t \bar{t}}=194 \pm 18 \text { (stat.) } \pm 46 \text { (syst.) pb }
$$

The leading uncertainties are from initial/final state radiation (ISR/FSR) (15\%) and event generator model uncertainty $(11 \%)$.

\section{Tau plus lepton channel at $\sqrt{s}=7 \mathrm{TeV}$}

The tau plus lepton channel consists of a final state with one $W$ boson decaying to a tau neutrino and tau lepton that decays hadronically, while the other $W$ decays to an electron or muon and neutrino. Measurements in this channel are motivated by searches for $t \rightarrow b H^{ \pm}\left(H^{ \pm} \rightarrow \tau^{ \pm} v_{\tau}\right)$. For this measurement [7], three or more jets with at least one

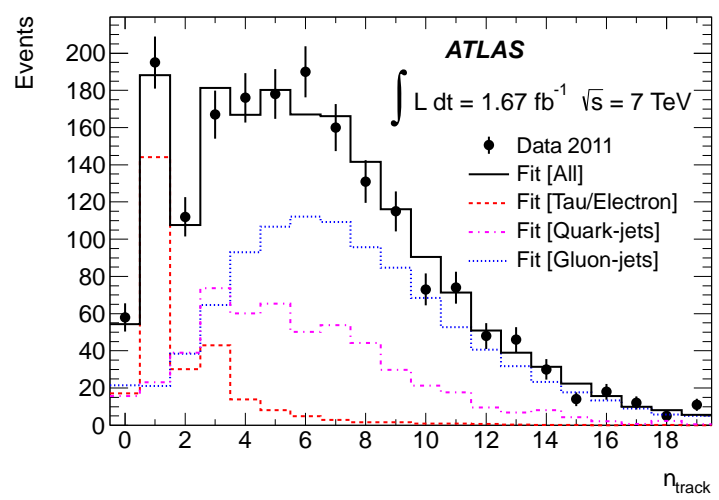

Figure 2. Distribution of track multiplicity for $\tau_{\text {had }}$ candidate events [6], showing the results of the likelihood fit (solid black line) to data (black points).

$b$-tag and one electron or muon are required, along with missing transverse momentum $E_{T}>30 \mathrm{GeV}$. Events are marked as same sign charge (SS) or opposite sign charge (OS) based on the reconstructed charge of the lepton and the tau candidate. Since tau candidates in the SS sample are almost entirely fake tau leptons and candidates in the OS sample are a mixture of fake and real, fake tau removal is achieved by subtracting SS events from the OS signal region. Since most processes contribute more to OS events than SS events due to correlation between the lepton charge and the leading quark charge, additional discriminating power between real and fake tau candidates is needed. A boosted decision tree $\left(\mathrm{BDT}_{j}\right)$ is employed to separate hadronic tau decays from jets in candidate events, yielding the distribution shown in Figure 3. The background template in this fit is derived from $0 b$-tag control sample after subtracting true tau events modeled from Monte Carlo simulation. The distribution of the output of $\mathrm{BDT}_{j}$ is fitted to extract the cross-section, that is measured to be

$$
\sigma_{t \bar{t}}=186 \pm 13 \text { (stat.) } \pm 20 \text { (syst.) } \pm 7 \text { (lumi.) } \mathrm{pb}
$$

\section{$5 t \bar{t}$ plus heavy flavor at $\sqrt{s}=7 \mathrm{TeV}$}

Top quark pairs can be produced in association with additional heavy flavor (HF) jets resulting from hadronization of $b$ - and $c$-quarks. The $t \bar{t}+b+X$ and $t \bar{t}+c+X$ final states are irreducible backgrounds for measurements of $t \bar{t}+H$ with $H \rightarrow b \bar{b}$, and are also important for studying new physics signatures coming from four-top final states or composite Higgs models. To study this process, we measure the ratio [8]

$$
\mathrm{R}_{\mathrm{HF}}=\frac{\sigma_{\mathrm{fid}}(t \bar{t}+\mathrm{HF})}{\sigma_{\mathrm{fid}}(t \bar{t}+j)}
$$

where $\sigma_{\text {fid }}(t \bar{t}+\mathrm{HF})$ is the fiducial cross-section measured from dilepton events with at least $3 b$-tagged jets, and $\sigma_{\text {fid }}(t \bar{t}+j)$ is the fiducial cross-section measured from dilepton events with at least 3 jets, 2 of which are $b$ tagged. The fiducial volume is defined to contain two 


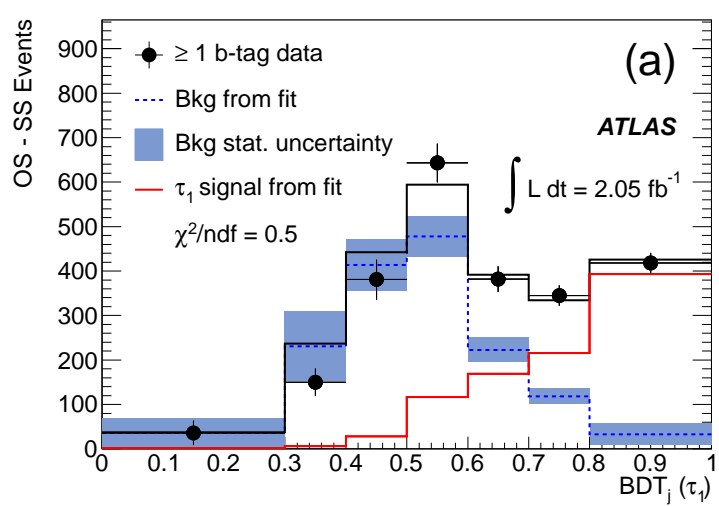

Figure 3. $\mathrm{BDT}_{j}$ (OS-SS) distributions of lepton plus single-track $\tau$ candidates [7]. The normalization of each template is derived from a fit to the data. The fitted contributions are shown as the light/red (signal), dashed/blue (background) and dark/black (total) lines. Shaded/blue bands are the statistical uncertainty of the background template.

charged leptons $(\ell=e, \mu)$ with transverse momentum $p_{T}(e)>25 \mathrm{GeV}$ or $p_{T}(\mu)>20 \mathrm{GeV}$ and pseudorapidity $|\eta(\ell)|<2.5$, and 3 or more jets with transverse momentum $p_{T}$ (jet) $>25 \mathrm{GeV}$ and pseudorapidity $\mid \eta($ jet $) \mid<2.5$. This measurement is performed using $4.7 \mathrm{fb}^{-1}$ of $\sqrt{s}=7 \mathrm{TeV}$ data. A maximum likelihood fit is employed to separate light and heavy flavor using two-dimensional templates binned in vertex mass and jet $p_{T}$, shown in Figure 4. The fit is performed in three mutually exclusive bins of $b$-jet purity whose boundaries are defined at operating points of $60,70,75 \% b$-jet efficiency. The dominant uncertainty in this analysis comes from the fiducial flavor composition $\mathrm{F}_{\mathrm{b} / \mathrm{HF}}$, which is defined as the fraction of $t \bar{t}+\mathrm{HF}$ events with additional $b$-quarks. Events with $b$-jets are reconstructed with a higher efficiency than events with c-jets, so the total reconstruction efficiency for $t \bar{t}+\mathrm{HF}$ events depends on how the HF is divided between $b$ - and $c$-jets. The variation of $R_{\mathrm{HF}}$ as a function of the fiducial flavor composition is shown in Figure 5. The ratio is measured to be

$$
\left.\mathrm{R}_{\mathrm{HF}}=[7.1 \pm 1.3 \text { (stat. })_{-2.0}^{+5.3} \text { (syst.) }\right] \%
$$

which is consistent at $1.4 \sigma$ with ALPGEN [9] and $0.6 \sigma$ with Powheg [10] Monte Carlo predictions.

\section{$6 t \bar{t}$ production with a veto on additional central jet activity}

The gap fraction is defined as

$$
f\left(Q_{0}\right)=\frac{n\left(Q_{0}\right)}{N}
$$

where $N$ is the number of selected events, and $n\left(Q_{0}\right)$ is the subset of events that do not contain an additional jet within a defined rapidity region with $p_{T}(\mathrm{jet})>Q_{0}$. A measurement of the gap fraction events in various rapidity regions is performed [11] on $2.05 \mathrm{fb}^{-1}$ of $\sqrt{s}=7 \mathrm{TeV}$ data. This measurement is motivated by its ability to significantly constrain $t \bar{t}$ modeling uncertainties and reduce their

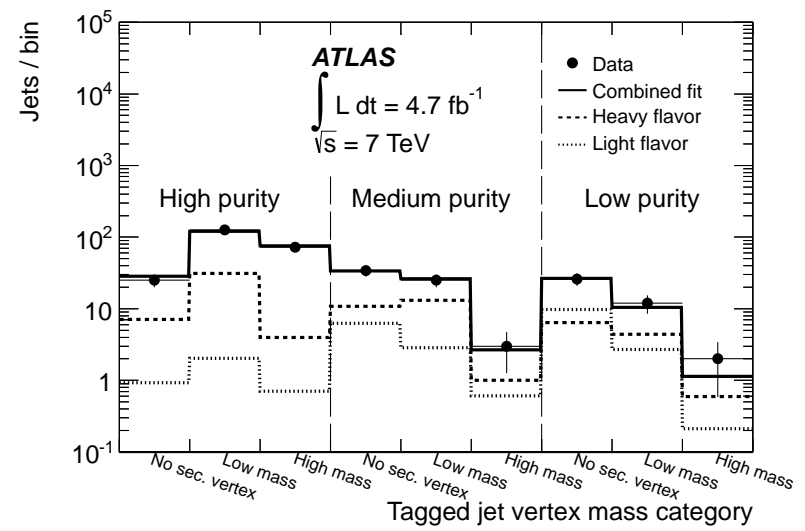

Figure 4. The result of the template fit (solid line) to the vertex mass distribution in data (points) [8]. The best fit is shown as a sum with separate contributions from heavy and light flavor.

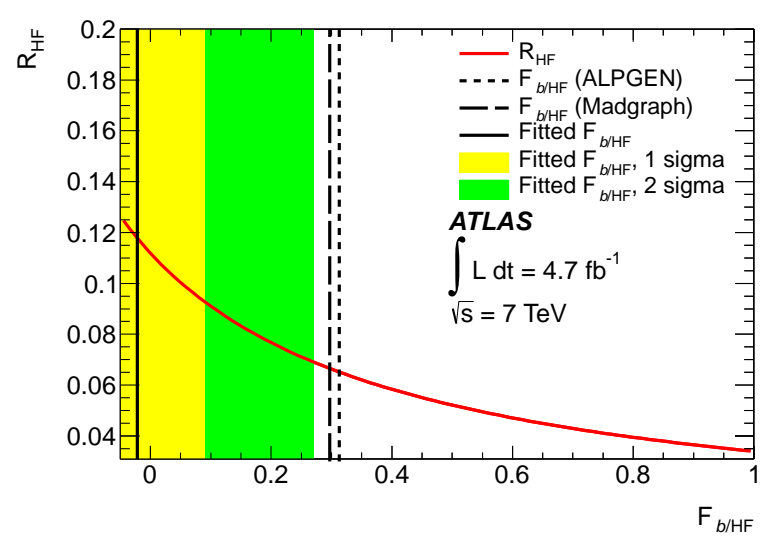

Figure 5. The ratio $\mathrm{R}_{\mathrm{HF}}$ of fiducial cross-sections as a function of $\mathrm{F}_{\mathrm{b} / \mathrm{HF}}$, the ratio of $t \bar{t}$ events with additional $b$-quarks to $t \bar{t}$ events with additional $c$ - or $b$-quarks [8]. $\mathrm{F}_{\mathrm{b} / \mathrm{HF}}$ as predicted in ALPGEN and Madgraph are indicated with vertical dashed lines. The fitted fraction of additional $b$-jets is used to extract $\mathrm{F}_{\mathrm{b} / \mathrm{HF}}$ (solid vertical line) from the data. The statistical uncertainty from the fit is used to define 1 sigma and 2 sigma uncertainty bands.

impact on precision top quark measurements. Events are chosen from the dilepton channel, requiring two oppositesign high- $p_{T}$ leptons $(e, \mu)$ and at least two $b$-tagged jets. Additional requirements are placed on dilepton invariant mass and missing transverse momentum $\left(\boldsymbol{E}_{T}\right)$ or $H_{T}$ (the scalar sum transverse momenta of visible physics objects) depending on the channel. The data are then corrected for detector effects to produce parton-level results. Due to the high purity of the sample (over $70 \%$ in each bin), a correction factor is derived for each bin to correct the reconstructed $Q_{0}$ distribution to a parton-level distribution. MC@ NLO [12], Alpgen, Sherpa [13], and Powheg Monte Carlo event generators are compared, and different settings for parameters controlling initial state radiation (ISR) are tested in samples simulated with AcERMC [14]. The study finds that each generator configuration predicts too much forward jet activity. In addition, MC@NLO is found to predict too little jet activity in the most central rapidity region. The findings of this analysis can be used to constrain the range of ISR uncertainties, as shown in Figure 6. 

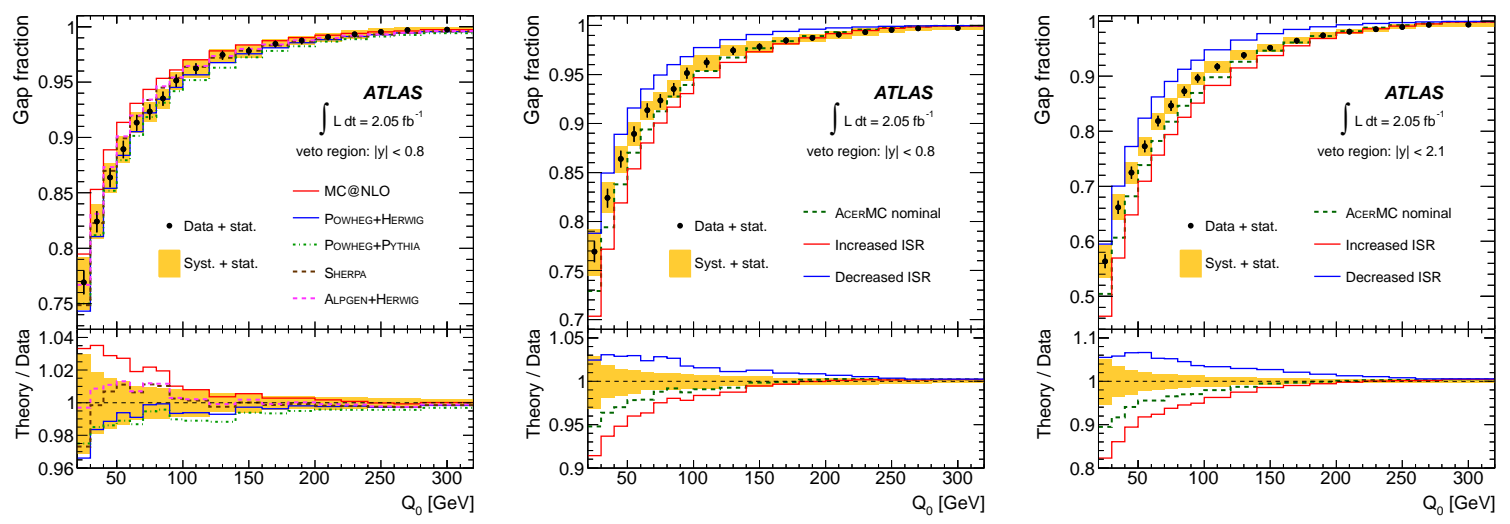

Figure 6. The measured gap fraction as a function of $Q_{0}$ for $|y|<0.8$ (left, center) and $|y|<2.1$ (right) [11]. The measurement is compared with the prediction from several generators (left) and from the ACERMC generator for different settings of the PYTHIA [18] parton shower parameters (center, right).

\section{Relative differential $t \bar{t}$ cross-section as a function of $m_{t \bar{t}}, p_{T, t \bar{t}}, y_{t \bar{t}}$}

A recent analysis [15] measures the differential $t \bar{t}$ crosssection, $\frac{1}{\sigma} \frac{\mathrm{d} \sigma}{\mathrm{d} X}$, with respect to three parton-level kinematic observables, $X=m_{t \bar{t}}, p_{T, t \bar{t}}$, and $y_{t \bar{t}}$, using $2.05 \mathrm{fb}^{-1}$ of data at $\sqrt{s}=7 \mathrm{TeV}$. These measurements provide a way to test the Standard Model at a more detailed level, especially in the $m_{t \bar{t}}$ distribution that can be sensitive to new physics contributions via $s$-channel $t \bar{t}$ production. Events are chosen from the lepton plus jets channel, with a cut on the kinematic likelihood assigned to events consistent with the $t \bar{t}$ hypothesis. Exactly one reconstructed electron or muon with transverse momentum $p_{T}(e)>25 \mathrm{GeV}, p_{T}(\mu)>$ $20 \mathrm{GeV}$ is required, with large $\mathbb{E}_{T}$ and $m_{T}(W)$ and at least four jets with transverse momentum $p_{T}>25$ (jet) $\mathrm{GeV}$, at least one of which is $b$-tagged. After subtracting backgrounds, distributions are unfolded from reconstructed quantities to parton-level quantities to make the crosssection measurements. This unfolding process uses a response matrix $\left(R_{i j}\right)$ derived from simulated $t \bar{t}$ events that describes the relation between reconstructed and partonlevel observables. The response matrix is inverted numerically to determine true differential cross-section distributions. The distributions found are in good agreement with the Standard Model, as shown in Figure 7.

\section{Jet multiplicity in $t \bar{t}$ events}

Differential cross-section measurements in bins of particle-jet multiplicity are performed using $4.7 \mathrm{fb}^{-1}$ of data at center of mass energy $\sqrt{s}=7 \mathrm{TeV}$ [16]. These measurements are important to understand and constrain uncertainties on initial and final state radiation in top events and test perturbative QCD. Measurement are performed with $N_{\text {jets }}$ binned in jet transverse momentum thresholds of 25, 40, 60 and $80 \mathrm{GeV}$. Events are chosen from the lepton plus jets channel, requiring a single electron or muon, with transverse momentum $p_{T}>$ $25(\ell) \mathrm{GeV}, E_{T}>30 \mathrm{GeV}, m_{T}(W)>35 \mathrm{GeV}$, and three or more jets with transverse momentum $p_{T}>25$ (jet) $\mathrm{GeV}$, at least one of which must be $b$-tagged. Backgrounds are subtracted and signal events are unfolded into the particlejet multiplicity distributions shown in Figure 8. Data are compard with predictions from several Monte Carlo generators, including AlPgEn+Pythia with variations on $\alpha_{S}$ constrained by Ref. [15]. The measurement is limited by systematic uncertainties. At low jet multiplicities, background model uncertainties are more significant and jet energy scale becomes the most significant source of uncertainty at high jet multiplicities. The data are found to disfavor the MC@NLO+HeRwIG [17] model, which predicts a lower jet multiplicity spectrum and generally softer jets.

\section{Summary}

In every inclusive, differential, and associated production measurement of $\sigma_{t \bar{t}}$, results agree well with latest SM theory predictions. ATLAS is testing Standard Model predictions involving the top quark with ever increasing precision using the full data sample collected in 2012 collisions at $\sqrt{s}=8 \mathrm{TeV}$.

\section{References}

[1] L. Evans and P. Bryant (editors) 2008 JINST 3 S08001.

[2] ATLAS Collaboration, 2008 JINST 3 S08003.

[3] M. Czakon, P. Fiedler, A. Mitov (2013), arxiv:1303.6254 [hep-ph].

[4] ATLAS Collaboration, ATLAS-CONF-2012-149, https://cds.cern.ch/record/1493488.

[5] ATLAS Collaboration, ATLAS-CONF-2012-097, https://cds.cern.ch/record/1460443.

[6] ATLAS Collaboration, Eur.Phys.J. C73, 2328 (2013).

[7] ATLAS Collaboration, Phys.Lett. B717, 89-108 (2012).

[8] ATLAS Collaboration, arXiv:1304.6386 [hep-ex]. 

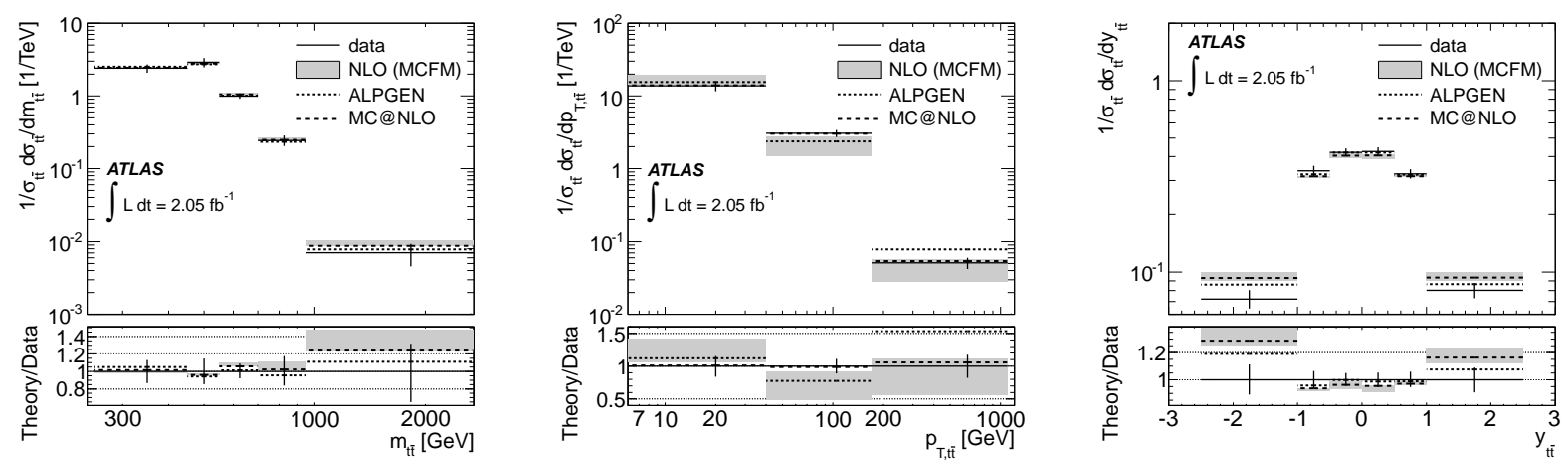

Figure 7. Relative differential cross-section versus $m_{t \bar{t}}$ (left), $p_{T, t \bar{t}}$ (center), and $y_{t \bar{t}}$ (right) [15]. Note that only the bin ranges along the $\mathrm{x}$-axis (and not the position of the vertical error bar) can be associated to the relative differential cross-section values on the $y$-axis. The relative cross-section in each bin shown in Table 3 is compared to the NLO prediction from MCFM [19]. The measured uncertainty represents $68 \%$ confidence level including both statistical and systematic uncertainties. The bands represent theory uncertainties. Predictions from MC@NLO and ALPGEN are shown for fixed settings of the generators' parameters.
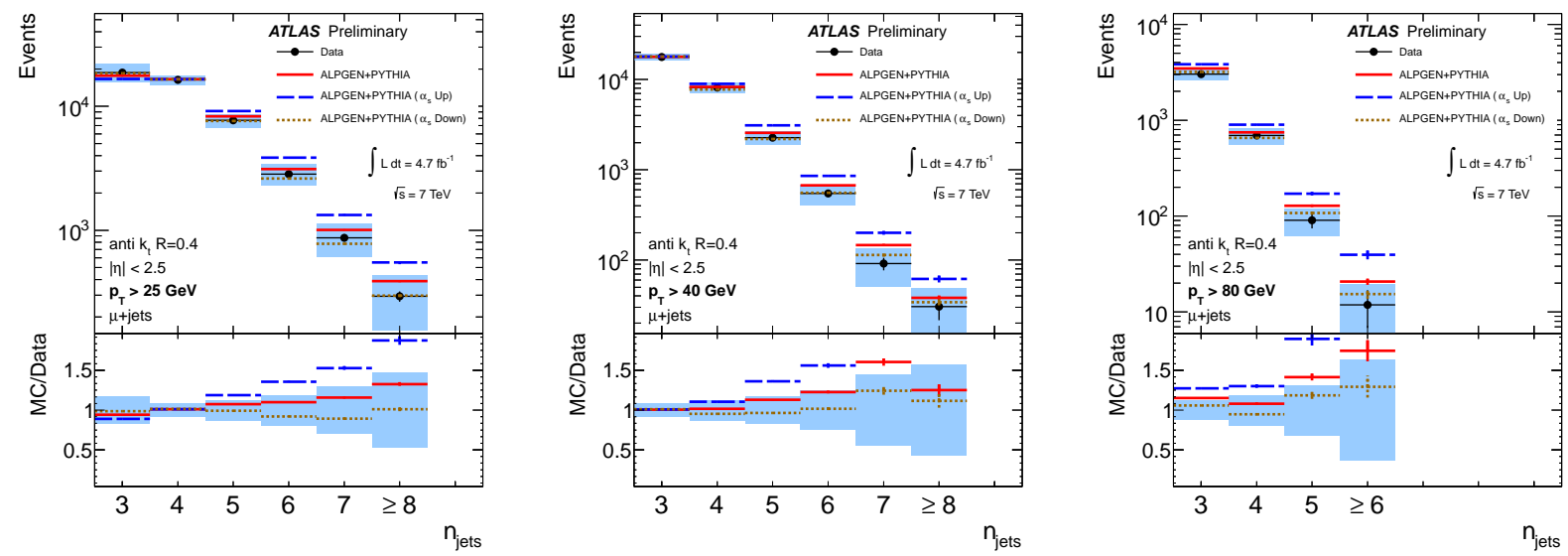

Figure 8. The particle-jet multiplicities for the muon channel and the jet $p_{T}$ thresholds 25 (left), 40 (center), and $80 \mathrm{GeV}$ (right) [16]. The data are shown in comparison with predictions from the Alpgen+Pythia and AlPgen+Pythia with varied $\alpha_{s}$ values. The data points and their corresponding statistical uncertainty are shown in black, whereas the total uncertainty (syst. + stat.) is shown as a shaded band. The Monte Carlo predictions are shown with their statistical uncertainties.

[9] M. Mangano et al., JHEP 07, 001 (2003).

[10] P. Nason, JHEP 11, 040 (2004).

[11] ATLAS Collaboration, Eur.Phys.J. C72, 2043 (2012).

[12] S. Frixione, B.R. Webber, JHEP 0206, 029 (2002).

[13] T. Gleisberg, et al., JHEP 0902, 007 (2009).

[14] B. Kersevan, E. Richter-Was (2004), arXiv:hep$\mathrm{ph} / 0405247$.
[15] ATLAS Collaboration, Eur.Phys.J. C73, 2261 (2013).

[16] ATLAS Collaboration, ATLAS-CONF-2012-155, https://cds.cern.ch/record/1493494.

[17] G. Corcella et al., JHEP 0101, 010 (2001).

[18] S. Mrenna, T. Sjostrand, P. Skands, JHEP 05, 0265 (2006)

[19] J. Campbell, R. Ellis, C. Williams, JHEP 1107, 018 (2011). 